\title{
A Novel Variant of the FZD4 Gene in a Chinese Family Causes Autosomal Dominant Familial Exudative Vitreoretinopathy
}

\author{
Lisha Yang ${ }^{a, b}$ Jiewen Fu ${ }^{a, c} \quad$ Jingliang Cheng ${ }^{a} \quad$ Chunli Wei $^{a} \quad$ Qi Zhou ${ }^{d}$ \\ Iqra ljaz ${ }^{\mathrm{a}}$ Hongbin $\mathrm{Lv}^{\mathrm{d}}$ Junjiang Fu ${ }^{\mathrm{a}, \mathrm{b}}$ \\ aKey Laboratory of Epigenetics and Oncology, the Research Center for Preclinical Medicine, Southwest \\ Medical University, Luzhou, Sichuan, 'Department of Obstetrics and Center for Prenatal Diagnosis, the \\ Affiliated Hospital of Southwest Medical University, Luzhou, Sichuan, Institute of Medical Technology, \\ Xiangtan Medicine and Health Vocational College, Xiangtan, Hunan, dDepartment of Ophthalmology, \\ Affiliated Hospital of Southwest Medical University, Luzhou, Sichuan, China
}

\section{Key Words}

Familial exudative vitreoretinopathy (FEVR) • FZD4 gene • Missense mutation • Targeted nextgeneration sequencing • Expression

\begin{abstract}
Background/Aims: Familial exudative vitreoretinopathy (FEVR) is a complex hereditary eye disorder characterized by incomplete development of the retinal vasculature, thereby affecting retinal angiogenesis. Methods: In this study, a Chinese autosomal dominant FEVR pedigree was recruited. Ophthalmic examinations were performed, targeted next-generation sequencing was used to identify the causative gene, and Sanger sequencing was conducted to verify the candidate mutation. Co-segregation analysis was performed to evaluate pathogenicity. Semiquantitative reverse transcription-PCR was applied to investigate the spatial and temporal expression patterns of the frizzled class receptor 4 (FZD4) gene in the mouse. Results: A novel heterozygous, deleterious variant of the FZD4 gene, c.A749G (p.Y250C), was identified in this FEVR pedigree, which co-segregated with the clinical phenotype. The amino acid tyrosine $(Y)$ is highly conserved both orthologously and paralogously. The FZD4 gene was highly expressed in the retina, sclera of the eye, ovary, kidney, and liver; ubiquitously expressed in other tissues; and highly expressed in 6 different developmental stages/times of retinal tissue. Conclusion: Our study is the first to identify that the novel heterozygous variant c.A749G (p.Y250C) in the FZD4 gene may be the disease-causing mutation in this FEVR family, extending its mutation spectrum. These findings further our understanding of the molecular pathogenesis of FEVR and will facilitate the development of methods for the diagnosis, prevention, and genetic counseling of this disease.

L. Yang, J. Fu and J. Cheng contributed equally to this work.




\section{Cellular Physiology Cell Physiol Biochem 2018;51:2445-2455 \begin{tabular}{ll|l} 
and Biochemistry & $\begin{array}{l}\text { DOl: 10.1159/000495901 } \\
\text { Published online: } 8 \text { December } 2018\end{array}$ & $\begin{array}{l}\text { O 2018 The Author(s). Published by S. Karger AG, Basel } \\
\text { www.karger.com/cpb }\end{array}$ \\
\cline { 2 - 3 }
\end{tabular} \\ Yang et al.: A Novel FZD4 Variant and FEVR}

\section{Introduction}

Familial exudative vitreoretinopathy (FEVR, OMIM: 133780) is a complex hereditary eye disorder characterized by the incomplete development of the retinal vasculature, particularly in pediatric groups, thereby affecting retinal angiogenesis [1-4]. The symptoms include avascular areas in the peripheral retina detectable by fluorescein angiography due to abnormal congenital retinal angiogenesis that can cause early-onset neovascularization, falciform folds, lipid exudation, and tractional retinal detachment [2]. The severity of its clinical manifestation is variable, ranging from the absence of visual impairment to total blindness. Its genotypic and phenotypic features are variable.

Currently, four genes involved in the norrin/frizzled signaling pathway (a variant of the Wnt signaling pathway) are linked to this disease: norrin disease protein (NDP, OMIM: 300658), frizzled class receptor (FZD4, OMIM: 604579), low-density lipoprotein receptorrelated protein 5 (LRP5, OMIM: 603506), and tetraspanin-12 (TSPAN12, OMIM: 613138), which are responsible for approximately $50 \%$ of FEVR cases [5]. In contrast, the genes encoding kinesin family member 11 (KIF11), RCC1 and BTB domain containing protein 1 (RCBTB1), zinc finger protein 408 (ZNF408), and catenin beta 1 (CTNNB1) are also responsible for a few cases of FEVR.

The relationships between the mutations in the causative genes in the norrin/frizzled signaling pathway and the resultant diseases are as follows: the NDP gene is responsible for X-linked recessive FEVR or Norrie disease, the FZD4 gene is responsible for autosomal dominant (AD) FEVR, the $L R P 5$ gene is responsible for AD or autosomal recessive (AR) FEVR, and the TSPAN12 gene is responsible for AD FEVR. In different studies of FEVR genes in Chinese pedigrees, the detection rates were variable; but did not exceed 50\% [6, 7]. Norrin/ frizzled signaling is critical for retinal angiogenesis by controlling retinal vascular growth and architecture both in developing eyes and in adult vasculature; impairment of this signaling pathway has profound effects on retinal vascular organization and leads to severe vascular abnormalities $[8,9]$.

Identification of the disease gene FZD4 for FEVR was first reported by Robitaille's group in 2002 [10]. In affected members of 2 unrelated families with AD FEVR, Robitaille et al. identified 2 different heterozygous deletions in exon 2 of the FZD 4 gene, resulting in the deletion of 2 conserved amino acids (namely Met493 and Trp494) or creation of a stop codon at residue 533 [10]. These mutations altered the seventh transmembrane domain and the intracellular carboxy-terminal tail, respectively.

The FZD4 gene (NM_012193.3), also known as frizzled 4 (7 transmembrane spanning receptor), frizzled family receptor 4, Fz-4, FzE4, HFz4, frizzled (Drosophila) homolog 4, frizzled homolog 4 (Drosophila), exudative vitreoretinopathy 1, and WNT receptor frizzled-4, is located on human chromosome 11q14.2; FZD4 protein (NP_036325.2) encodes a deduced 537 -amino acid protein. This gene is a member of the frizzled gene family. Members of this family encode 7-transmembrane domain proteins, which are receptors for the wingless-type MMTV integration site family of signaling molecules. Gene ontology (GO) annotations of this gene revealed G-protein coupled receptor (GPCR) activity and protein heterodimerization activity. Most frizzled receptors are coupled with $\beta$-catenin canonical signaling and may play a role in the positive regulation of the $W n t / \beta$-catenin signaling pathway.

The presentation and course of FEVR shows high variability, making its diagnosis and management challenging. FZD4 mutations in patients with AD FEVR have not been well described in the Chinese population. Here, targeted next-generation sequencing technology, the most available land promising method [11-18] for combined bioinformatics and expression profile analysis, was applied to identify a novel, heterozygous variant of the FZD4 gene in a Chinese family with AD FEVR. 


\section{Cellular Physiology Cell Physiol Biochem 2018;51:2445-2455 and Biochemistry \begin{tabular}{l|l} 
DOI: 10.1159/000495901 & $\begin{array}{l}\text { C } 2018 \text { The Author(s). Published by S. Karger AG, Basel } \\
\text { www.karger.com/cpb }\end{array}$
\end{tabular} \\ Yang et al:: A Novel FZD4 Variant and FEVR}

\section{Materials and Methods}

\section{Ethics statement}

The Ethics Committee of Southwest Medical University approved this study. Written informed consent was obtained from the participants or their guardians, in accordance with the guidelines of the Declaration of Helsinki (1983 Revision).

\section{Pedigree, proband, and clinical assessment}

The pedigree (M362) consisted of a proband (Fig. 1, pedigree II: 2, arrow), the proband's father with the same disease, a normal mother, and a normal elder sister in a Chinese family from Yibin, a city in Sichuan Province in the southwest of China. For clinical assessment, a clinical history and ophthalmic examinations were performed with the proband, including best-corrected Snellen visual acuity, Humphrey visual fields, slit-lamp biomicroscopy, fundoscopy, [ultra-wide-field fundus photographs (FPs), and ultra-wide-field fundus fluorescent photographs (FFPs) [19].

\section{Blood sampling and DNA extraction}

Fresh peripheral blood samples $(2 \mathrm{~mL})$ were taken and genomic DNA (gDNA) was extracted from the proband and pedigree members using a phenol/chloroform method [20,21]. Blood samples were also taken from 100 ethnically matched and healthy control volunteers without any disease history.

\section{Capture panel design and target sequencing}

To identify the disease-causing gene and to identify mutation, TES analyses were performed on the gDNA sample of the proband from pedigree M362 DNA, according to the instructions for Illumina pairedend libraries (Illumina, Inc., San Diego, CA) as reported previously [11, 12, 14]. The capture Agilent probes were used as in previously published studies $[11,12,14,22]$ with a retinal disease capture panel that included FEVR-related genes: NDP, FZD4, LRP5, TSPAN12, ZNF408, CTNNB1, RCBTB1, and KIF11. In brief, $2 \mu \mathrm{g}$ of the extracted proband gDNA was randomly sheared by sonication into $300-500 \mathrm{bp}$ fragments. The 5 '-ends of the DNA fragments were phosphorylated by polynucleotide kinase, and adenine was added at the $3^{\prime}$-ends. Hybridization to the pre-capture libraries was quantified and applied for sequencing on an Illumina HiSeq 2000 (Illumina, Inc.), according to the manufacturer's protocols [5].

\section{Data analysis}

Paired-end sequencing Illumina reads were aligned to the human hg19 reference genome using Burrows-Wheeler Aligner version 0.6.1 and the public online University of California Santa Cruz (UCSC) database (http://genome.ucsc.edu/) [23]. Variations in single nucleotide polymorphisms (SNPs) and insertions/deletions were refined using Atlas-SNP2 and Atlas-Indel2 [24]. Variant frequency data were applied to the CHARGE consortium [25], human 1000 Genomes [26], ANNOVAR [27], ESP-6500 [28], and ExAC databases to look for pathogenic mutations in all candidate genes. Only very rare variants were considered, with a dominant frequency cutoff of $0.05 \%$ [5]. Sequencing depth 4 , estimated copy number 2 , SNP quality 20 , and a distance between two SNPs of $>5$ were used as the filtration criteria for candidate SNPs, according to previously reported studies $[29,30]$. Sequence variants should not be annotated in any of the above public databases. The pathogenicity of each variant was assessed with the following databases: PolyPhen-2 (http://genetics.bwh.harvard.edu/pph2/), Sorting Intolerant From Tolerant (SIFT) (http:// sift.jcvi.org/www/SIFT_enst_submit.html), MutationTaster (http://www.mutationtaster.org/), and I-Mutant2.0 (http://folding.biofold.org/i-mutant/i-mutant2.0.html). Variants, considered as pathogenic candidates, were searched for in the Human Gene Mutation Database (HGMD) (http://www.hgmd.cf.ac. uk/ac/gene.php?gene=FZD4) to determine whether they had been reported previously.

\section{Primer design and PCR amplification}

For mutation verification and co-segregation analysis, polymerase chain reaction (PCR) amplification and direct Sanger sequencing" of the variant were applied to gDNA of all available individuals [29, 30]. A locus-specific primer pair (M362-FZD4) was designed using the online Primer3 program (http://primer3. ut.ee/) with gDNA sequences containing the identified c.A749G mutation in FZD4 (Table 1). A product of 285 bp was amplified using gDNA as the template. 
Table 1. PCR primers and PCR product sizes

\begin{tabular}{|c|c|c|c|c|c|}
\hline Primer name & Left primer & Sequence $\left(5^{\prime}-3^{\prime}\right)$ & Right primer & Sequence $\left(5^{\prime}-3^{\prime}\right)$ & Size ${ }^{\circ} \mathrm{C}$ \\
\hline M362-FZD4 & M362-FZD4-7491 & aggagcctgaactgtgtgct & 1362-FZD4-749 & 2 aggttc & 28560 \\
\hline T-fzd4 & $T-f$ & $\mathrm{acc}$ & RT-fzd4-1 & ggcaaacccaaattctct & 5260 \\
\hline T-b-actin-m & RT-b-actin-mL & tgttaccaactgggacgaca & RT-b-actin-mR & tctcagctgtggtggtgaa & g 39260 \\
\hline
\end{tabular}

Dideoxy DNA sequencing and co-segregation analysis

The PCR products were sequenced using the dideoxy DNA sequencing method on an ABI-3500DX sequencer (Applied Biosystems, Inc., Foster City, CA) in our laboratory using a specific primer (M362-FZD4-749L) listed in Table 1. All unrelated, ethnically matched controls were sequenced using the aforementioned primer. Co-segregation analysis was performed based on the DNA sequencing results.

\section{Protein structure and bioinformatics analysis}

A search for conserved domains within a protein or coding nucleotide sequence using the input FZD4 was performed through the online NCBI Conserved Domains Database (https://www.ncbi.nlm.nih.gov/Structure/cdd/ wrpsb.cgi) [31, 32]. Homologs of the FZD4 gene were identified using the online NCBI HomoloGene system: (https://www.ncbi.nlm.nih.gov/homologene?Db=homol ogene\&Cmd=Retrieve\&list_uids=7325). Protein sequence alignment of human FZD4 with homologs from the human frizzled protein family was performed using the multiple sequence alignment CLUSTALW program (https://www. genome.jp/tools-bin/clustalw) with the following Homo sapiens protein sequences: FZD1 (NP_003496.1), FZD2 (NP_001457.1), FZD3 (NP_059108.1), FZD4 (NP_036325.2), FZD5 (NP_003459.2), FZD6 (NP_001158087.1), FZD7 (NP_003498.1), FZD8 (NP_114072.1), FZD9 (NP_003499.1), and FZD10 (NP_009128.1).

RNA extraction and reverse transcription (RT)- PCR

Total RNA was extracted from the mice at the indicated ages and the indicated tissues using an RNA kit according to our previously reported standard protocols [30]. RNA quality was measured using a NanoDrop 2000 spectrophotometer (Thermo Fisher Scientific, Wilmington, DE) and 1.5\% agarose gel electrophoresis. cDNA synthesis was performed using random oligomer primers and a reverse transcriptase kit [30]. The RT-primer sequences, product size, and PCR conditions (annealing temperature) are listed in Table 1. Semiquantitative RT-PCR was performed using the primer pair RT-fzd4 for the mouse Fzd4 gene; the ß-actin gene of mouse was used as an internal control and amplified using the primer pair RT-b-actin-mL and RT-b-actin$\mathrm{mR}$ [30] (Table 1). Each RT-PCR assay was done in three independent tests for verification.

\section{Results}

\section{Pedigree and clinical characteristics}

The proband (Fig. 1, II: 2) was a 7-year-old boy from a non-consanguineous Chinese family who was born at full term by cesarean section, was found to have low vision at 3 years of age, and was admitted to hospital due to a further reduction in his visual acuity at 7 years of age. Ultra-wide-field FPs and FFPs of both eyes of the proband are shown in Fig. 2. Fundus examination revealed avascularity in the peripheral retina in both eyes but no 


\section{Cellular Physiology Cell Physiol Biochem 2018;51:2445-2455

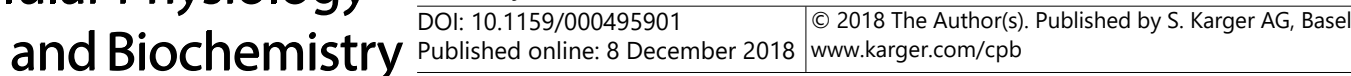 Yang et al.: A Novel FZD4 Variant and FEVR}

neovascularization (Fig. 2A\&B). FFPs clearly demonstrated peripheral retinal avascularity. Abnormal vascular patterns including venous-venous looping, new vessels at the junction, brushed peripheral retinal vessels, and proliferation of branching vessels were observed; superotemporal retinal fold formation and the absence of the macula in the right eye were also noticed (Fig. 2C\&D). Optometry vision analysis revealed myopia (1000 and 600 degrees in the right and left eye, respectively). The proband's father had the same retinal disease when he was a child, but his vision was better than that of the proband at the age of 32 years, suggesting an $\mathrm{AD}$ inheritance. As a result, the proband was categorized as stage I FEVR according to Trese's staging system.

\section{Next-generation sequencing analysis and putative pathogenic mutation screening}

To determine the disease-causing gene mutation, targeted capture high-throughput sequencing of 195 retinal disease-causing genes was performed successfully using a capture panel with the gDNA sample of the proband (Fig. 1, pedigree II: 2). A heterozygous, missense variant (c.A749G) with a SNP of exon 2 in the FZD4 gene (NM_012193.3) was identified in this proband, leading to an amino acid change from tyrosine (Tyr, Y) to cysteine (Cys, C) at position 749 (p.Y250C) of the FZD4 protein (NP_036325.2) (Fig. 1 II: 2). PolyPhen-2 analysis showed probable damage for this change (score 1); MutationTaster revealed the change to be disease causing (score 0.9999999999659); SIFT indicated it was damaging (score 0); and
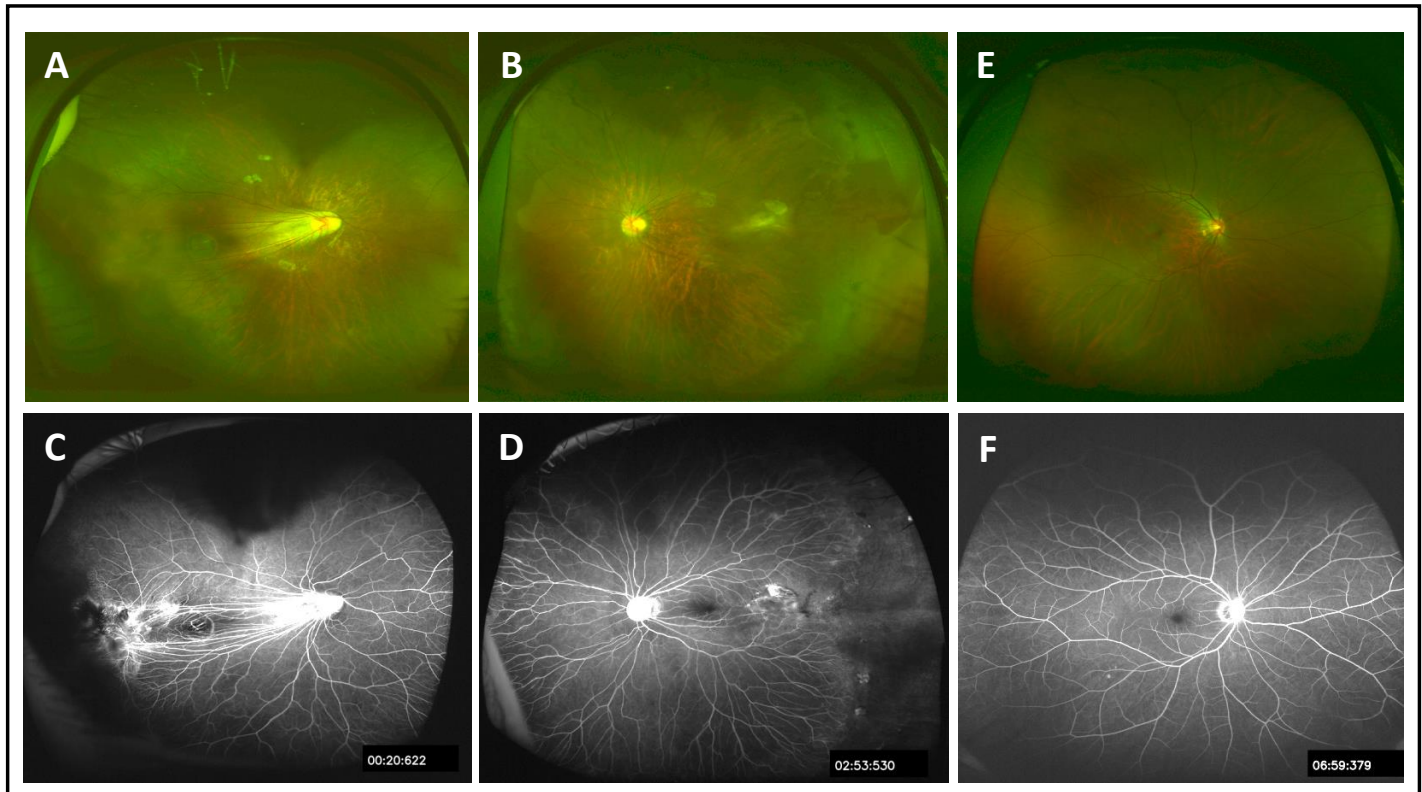

Fig. 2. Representatively ultra-wide-field FPs and FFPs of patient II: 2 from both eyes. A\&B. FPs from the proband (right and left eye, respectively). C\&D. FFPs from the proband (right and left eye, respectively). E\&F. FPs and FFPs in a normal control, respectively.

Table 2. Characteristics of the FZD4 variant in the proband and analysis of its disease-causing effects. Abbreviations: SIFT, Sorting Intolerant From Tolerant; FZD4, frizzled class receptor 4; c, variation at the cDNA level; p, variation at the protein level; Y250C, tyrosine substitution conserved cysteine at codon 250; Hetero, heterozygote; PD, probably damaging; DC, disease causing; DDG, free energy change value (DDG $>0$ : increased stability); D, damaging.* All nucleotides and amino acids are abbreviated according to the International Union of Pure and Applied Chemistry (IUPAC)

\begin{tabular}{|c|c|c|c|c|c|c|c|c|}
\hline \multirow{2}{*}{ Gene Exon } & \multicolumn{4}{|c|}{ Variation } & \multirow{2}{*}{ Polyphen-2 } & \multirow{2}{*}{ MutationTaster } & \multirow{2}{*}{ I-Mutant2.0 } & \multirow{2}{*}{ SIFT ExAC } \\
\hline & & * Protein* & Type & Status & & & & \\
\hline ZD4 2 & c.A749G & p.Y250C N & Missens & Hetero & PD (1) & C (0.999999999965 & DG $(0.75 \mathrm{kcal} / \mathrm{m}$ & D (0) Novel \\
\hline
\end{tabular}


I-Mutant2.0 for the free energy change value indicated increased stability (DDG $=0.75 \mathrm{kcal} /$ $\mathrm{mol},>0$ ). The deleterious and pathogenic aspects of the FZD4 c.A749G: p.Y250C mutation are presented in Table 2. Thus, this missense variant c.A749G (p.Y250C) in the FZD4 gene most likely damaged protein function in this Chinese FEVR family. This variant was determined to be a novel mutation after screening the ExAC and HGMD databases (Table 2).

\section{Mutation verification and segregation analysis}

Confirmation of the mutation and segregation analysis were performed by dideoxy DNA sequencing (Fig. 3). The c.A749G variant of FZD4 was confirmed to be heterozygous in the proband (pedigree II: 2; Fig. 3A), and we identified the heterozygous mutation in the proband's father with FEVR (pedigree I: 1; Fig. 3B), while the proband's mother and elder sister had the wild-type gene and a normal phenotype (pedigree I: 2, II: 1; Fig. 3C \& data not shown). Thus, it was demonstrated that the c.A749G variant in the FZD4 gene cosegregated with the disease phenotype in the family members that were tested. This mutant was absent in 100 normal, ethnically matched controls (data not shown). All together, these findings show complete co-segregation| of the mutation in the pedigree of the FEVR family and pinpoint its role in pathogenesis of this disease.

\section{Functional effects of the pathogenic variant c.A749G (p.Y250C) for FZD4}

Searching through the Conserved Domains Database in NCBI revealed that FZD4 has three conserved domains (Fig. 4A), namely Frizzled/Smoothened family membrane region (frizzled, pfam01534), Fz (frizzled) domain (CRD_FZ, cl02447), and the 7 transmembrane receptor domain (7tm_2, cl19289) [31]. The p.Y250C mutation is located in the 7tm_2 domain (secretin family) (Fig. 4A). This family is known as family B, the secretin-receptor family, or family 2 of GPCRs. Three distinct sub-families, namley B1, B2, and B3, were recognized. Subfamily B1 contains classical hormone receptors that are involved in cAMPmediated pathways. Subfamily B2 contains receptors with long extracellular N-termini, such as CD97, calcium-independent receptors for latrotoxin, and brain-specific angiogenesis inhibitors. Subfamily B3 includes Methuselah and other Drosophila proteins. Other than the typical 7-transmembrane region, characteristic structures include an amino-terminal extracellular domain involved in ligand binding, and an intracellular loop (IC3) required for specific G-protein coupling. Orthologous comparison of $H$. sapiens FZD4 to nine other species (Pan troglodytes, Macaca mulatta, Canis lupus, Bos taurus, Mus musculus, Rattus norvegicus, Gallus gallus, Xenopus tropicalis, and Danio rerio) indicated that tyrosine (Y) is highly conserved (Fig. 4B). Paralogous comparison of FZD4 to other family members in $H$. sapiens, including FZD1-3, 5-10, indicated that this tyrosine (Y) is also highly conserved (Fig. 4C). Comprehensively, this study showed that the FZD4 heterozygous mutation, c.A749G (p.Y250C), causes FEVR disease.

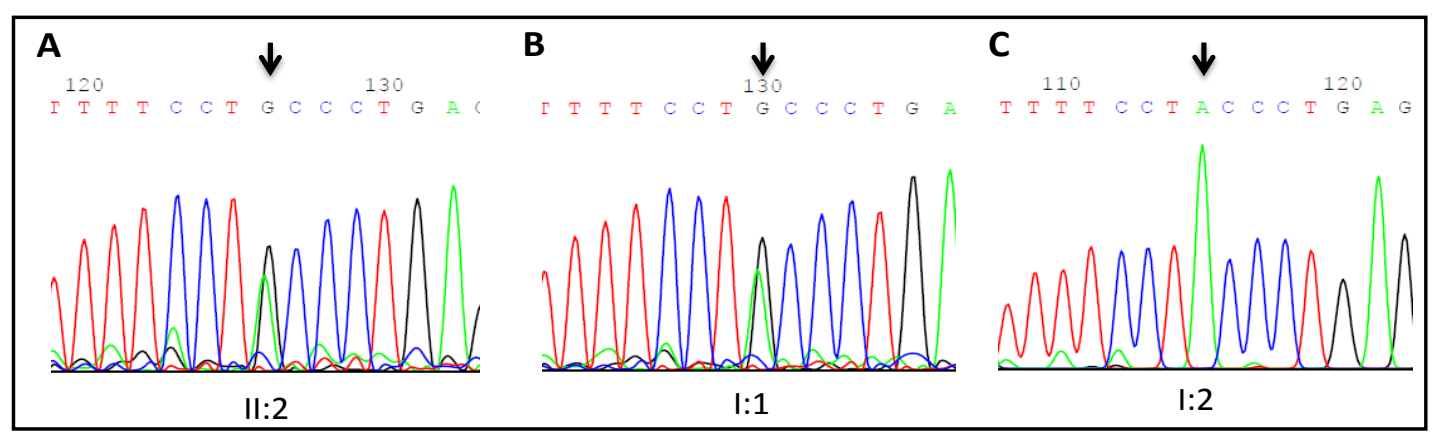

Fig. 3. Pyrogram profiles for mutation verification by dideoxy DNA sequencing. A-C indicate the sequencing results in II: 2 (heterozygous mutant), I: 1 (heterozygous mutant), I: 2 (wild-type), respectively. The arrows indicate the mutation at the nucleotide position NM_012193.3: exon 2: c.A749G in the FZD4 gene. 


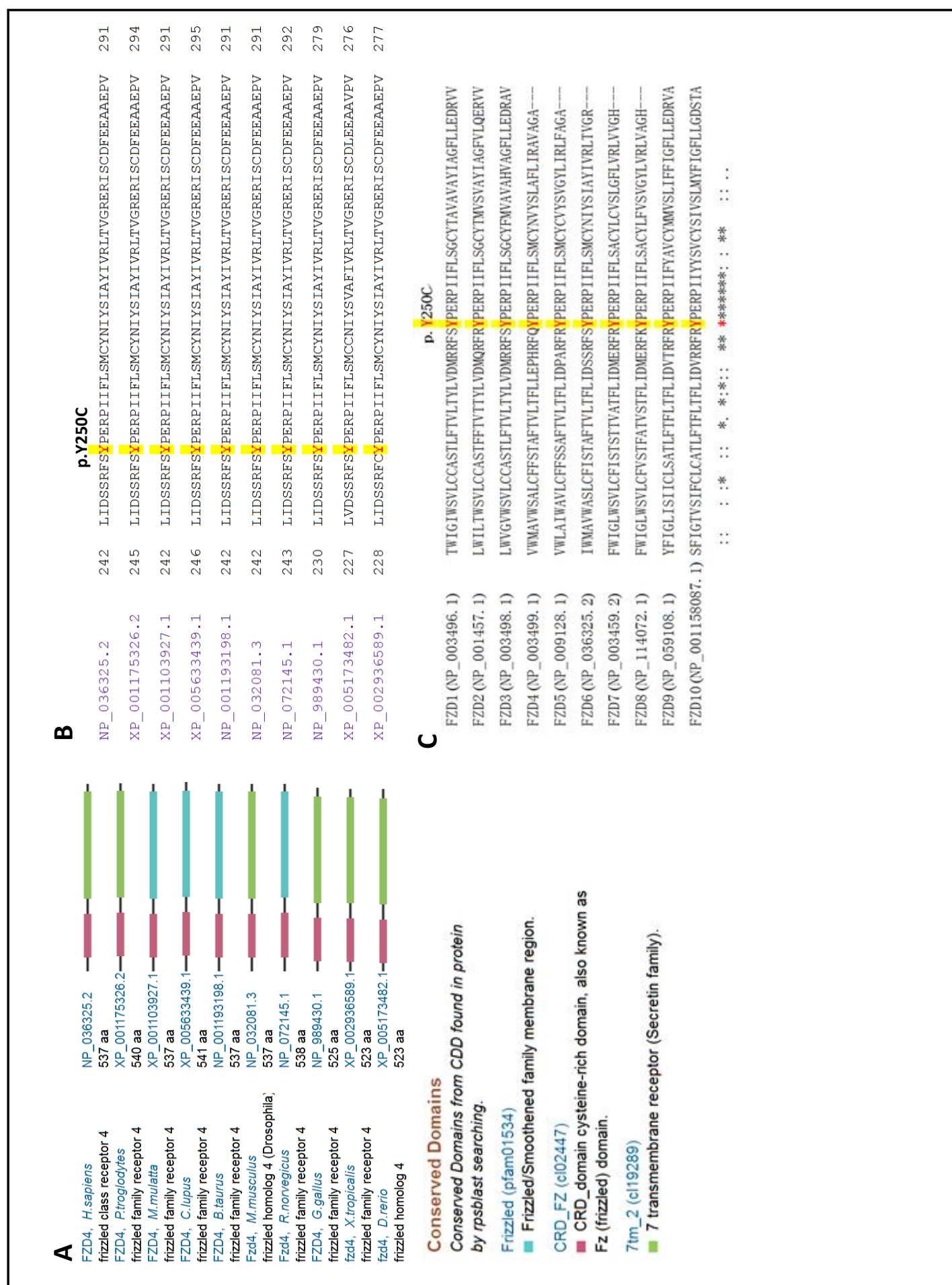

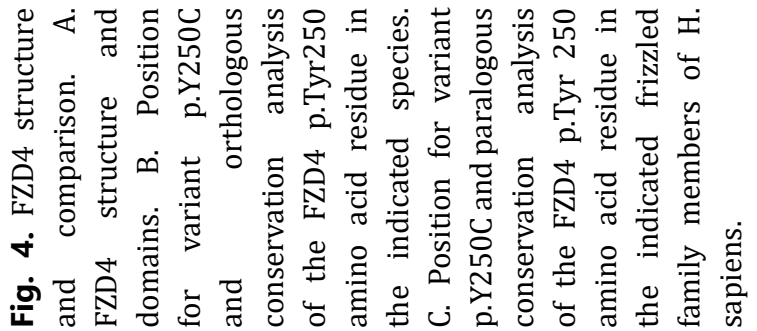




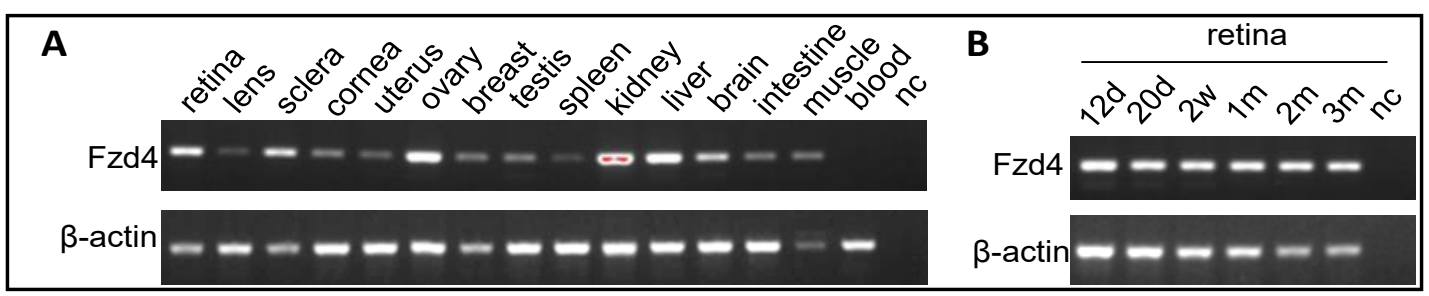

Fig. 5. Expression of Fzd4 mRNA in the indicated tissues (A) and at the indicated development stages or times in retinal tissue (B) in mice. d, day(s); w, week(s); m, month(s); nc, negative control without any template DNA; muscle, skeletal muscle. Whole eye balls from embryos at 12.5 days (12d) and 20.5 days (20d) in panel B, respectively.

\section{Fzd4 mRNA expression profiles}

The expression of Fzd4 mRNA in the indicated 15 different tissues and at the indicated 6 different developmental stages of the retina was investigated in mice (Fig. 5). The results showed that Fzd4 transcripts were highly expressed in the retina and sclera of the eye, ovary, kidney, liver, and brain; were weakly expressed in the lens, cornea, uterus, breast, testis, spleen, intestine, and skeletal muscle; had no detectable expression in blood (Fig. 5A); and were highly expressed at the 6 different developmental stages/times of retinal tissue (Fig. 5B). The very high expression of $\mathrm{Fzd} 4$ in the retina and its ubiquitous expression in other tissues and organisms indicated that FZD4 plays important roles in the functions of retina/ eye and other tissues in Wnt signaling pathways for angiogenesis.

\section{Discussion}

As FEVR progresses, retinal neovascularization, subretinal exudation, and partial and total retinal detachment may occur, which are symptoms that may be associated with certain mutations. With early diagnosis and prompt management, visual loss can be prevented with laser photocoagulation and anti-VEGF injections. Laser treatment or cryotherapy has been reported in patients with FEVR [33]. In the case of retinal detachment, pars plana vitrectomy alone or combined with scleral buckling should be considered. Vitrectomy with focal removal and segmentation of the fibrovascular tissue in the posterior pole might be a good surgical option in eyes with FEVR [34]. Wide-field imaging with fluorescein angiography is crucial in the management of this disease. Wide-field retinal imaging is also helpful for detecting asymptomatic family members of index patients. The determination of asymptomatic family members with the disease at child-bearing age is essential for genetic counseling and neonatal screening. Earlier diagnosis and management results in a better prognosis [33]. However, mutations of FZD4 in patients with AD FEVR have not been well described in the Chinese population by next-generation sequencing-based genetic diagnosis [35]. In this study, we identified a heterozygous, deleterious variant of the FZD4 gene, c.A749G (p.Y250C), in a Chinese family, which led to the disease. After searching HGMD (access date, May 18, 2018), only 48 pathogenic variants were found to have been reported, including missense/nonsense (48), small deletions (8), a small insertion (1) and a complex rearrangement (1). To the best of our knowledge, FZD4 variant c.A749G (p.Y250C) is a novel mutation, thereby extending its mutation spectrum. The proband was categorized as stage I FEVR; thus, we explored the genotype-phenotype correlations by linking FEVR stage I with this FZD4 variant.

These findings showed that the FZD4 mutation c.A749G (p.Y250C) causes FEVR in the Chinese pedigree of our study. The identification of FZD4 mutations in FEVR patients, which showed the highest incident rate of this disease in Chinese populations, will not only help in neonatal screening but also in family planning and accurate prenatal gene diagnosis, which may facilitate genetic counseling and prevent the birth of babies with FEVR in undeveloped and developing countries. 
GO annotations related to the FZD4 gene revealed GPCR activity and protein heterodimerization activity. An FZD4 mutant with a frameshift at Leu501 does not accumulate at the plasma membrane, but is trapped in the endoplasmic reticulum [36]. Through heterodimerization, this mutant FZD4 could trap wild-type FZD4 and inhibit its signaling pathways. Xu et al. found that NDP and FZD4 function as a ligand-receptor in vascular phenotypes caused by NDP and FZD4 mutations in humans and mice. The specificity and high affinity of NDP-FZD4 binding and its high efficiency with NDP induce FZD4- and LRP-dependent activation of Wnt signaling [37]. This NDP-FZD4 signaling system plays a vital role in vascular development of both the eye and ear. NDP is a potent trigger of FZD4 ubiquitination and induces the internalization of the NDP receptor complex into the endolysosomal compartment [38]. I-Mutant2.0 analysis of the free energy change value (DDG>0) of the FZD4 p.Y250C mutant protein indicated an increase in its stability. In this regard, this FZD4 mutation might affect its heterodimerization, trapping wild-type FZD4 and inhibiting its signaling, thus explaining the genetic dominance of the FEVR allele studied in our pedigree.

The amino acid tyrosine (Y) is highly conserved orthologously in the frizzled 4 protein of multiple species and is highly conserved paralogously in the frizzled family of H.sapiens. In addition, the Fzd4 gene is highly expressed in the retina and is ubiquitously expressed in different tissues, as well as at the different developmental stages/times of the retina we tested, indicating that FZD4 plays an important role in angiogenesis in the human retina/ eye, as well as in that of other organisms.

\section{Conclusion}

In conclusion, our study is the first to identify that the novel heterozygous variant c.A749G (p.Y250C) in the FZD4 gene is the disease-causing mutation for FEVR in our Chinese pedigree, extending its mutation spectrum. Targeted next-generation sequencing technology provides a useful and cost-effective genetic method for molecular diagnosis [30, 39]. These findings also enhance our understanding of genotype-phenotype correlations in FEVR for diagnosis, prognosis, prevention, and genetic counseling.

\section{Acknowledgements}

The authors thank the patients and their family for supporting this project. We thank Dr. Rui Chen at Baylor College of Medicine for support with next-generation sequencing. We thank Ms. Shangyi Fu at the University of Houston/Baylor College of Medicine for final English editing of the manuscript. This work was supported by the National Natural Science Foundation of China (30371493, 31701087, and 81672887).

\section{Disclosure Statement}

The authors declare no conflicts of interest.

\section{References}

1 Canny CL, Oliver GL: Fluorescein angiographic findings in familial exudative vitreoretinopathy. Arch Ophthalmol 1976;94:1114-1120.

-2 Iarossi G, Bertelli M, Maltese PE, Gusson E, Marchini G, Bruson A, Benedetti S, Volpetti S, Catena G, Buzzonetti L, Ziccardi L: Corrigendum to "Genotype-Phenotype Characterization of Novel Variants in Six Italian Patients with Familial Exudative Vitreoretinopathy”. J Ophthalmol 2017;2017:7969364. 


\section{Cellular Physiology Cell Physiol Biochem 2018;51:2445-2455 \begin{tabular}{l|l|l} 
and Biochemistry Published online: 8 December 2018 & $\begin{array}{l}\text { (c) } 2018 \text { The Author(s). Published by S. Karger AG, Basel } \\
\text { www.karger.com/cpb }\end{array}$ \\
\hline
\end{tabular}}

Yang et al.: A Novel FZD4 Variant and FEVR

3 Gilmour DF: Familial exudative vitreoretinopathy and related retinopathies. Eye 2015;29:1-14.

4 Criswick VG, Schepens CL: Familial exudative vitreoretinopathy. Am J Ophthalmol 1969;68:578-594.

5 Salvo J, Lyubasyuk V, Xu M, Wang H, Wang F, Nguyen D, Wang K, Luo H, Wen C, Shi C, Lin D, Zhang K, Chen R: Next-generation sequencing and novel variant determination in a cohort of 92 familial exudative vitreoretinopathy patients. Invest Ophthalmol Vis Sci 2015;56:1937-1946.

6 Rao FQ, Cai XB, Cheng FF, Cheng W, Fang XL, Li N, Huang XF, Li LH, Jin ZB: Mutations in LRP5,FZD4, TSPAN12, NDP, ZNF408, or KIF11 Genes Account for 38.7\% of Chinese Patients With Familial Exudative Vitreoretinopathy. Invest Ophthalmol Vis Sci 2017;58:2623-2629.

7 Tang M, Sun L, Hu A, Yuan M, Yang Y, Peng X, Ding X: Mutation Spectrum of the LRP5, NDP, and TSPAN12 Genes in Chinese Patients With Familial Exudative Vitreoretinopathy. Invest Ophthalmol Vis Sci 2017;58:5949-5957.

-8 Ye X, Wang Y, Cahill H, Yu M, Badea TC, Smallwood PM, Peachey NS, Nathans J: Norrin, frizzled-4, and Lrp5 signaling in endothelial cells controls a genetic program for retinal vascularization. Cell 2009;139:285298.

-9 Masckauchan TN, Kitajewski J: Wnt/Frizzled signaling in the vasculature: new angiogenic factors in sight. Physiology 2006;21:181-188.

10 Robitaille J, MacDonald ML, Kaykas A, Sheldahl LC, Zeisler J, Dube MP, Zhang LH, Singaraja RR, Guernsey DL, Zheng B, Siebert LF, Hoskin-Mott A, Trese MT, Pimstone SN, Shastry BS, Moon RT, Hayden MR, Goldberg YP, Samuels ME: Mutant frizzled-4 disrupts retinal angiogenesis in familial exudative vitreoretinopathy. Nat Genet 2002;32:326-330.

11 Zhu L, Cheng J, Zhou B, Wei C, Yang W, Jiang D, Ijaz I, Tan X, Chen R, Fu J: Diagnosis for choroideremia in a large Chinese pedigree by nextgeneration sequencing (NGS) and noninvasive prenatal testing (NIPT). Mol Med Rep 2017;15:1157-1164.

12 Wang F, Wang H, Tuan HF, Nguyen DH, Sun V, Keser V, Bowne SJ, Sullivan LS, Luo H, Zhao L, Wang X, Zaneveld JE, Salvo JS, Siddiqui S, Mao L, Wheaton DK, Birch DG, Branham KE, Heckenlively JR, Wen C, Flagg K, Ferreyra H, Pei J, Khan A, Ren H, Wang K, Lopez I, Qamar R, Zenteno JC, Ayala-Ramirez R, Buentello-Volante B, Fu Q, Simpson DA, Li Y, Sui R, Silvestri G, Daiger SP, Koenekoop RK, Zhang K, Chen R: Next generation sequencing-based molecular diagnosis of retinitis pigmentosa: identification of a novel genotype-phenotype correlation and clinical refinements. Hum Genet 2014;133:331-345.

13 Yohe S, Thyagarajan B: Review of Clinical Next-Generation Sequencing. Arch Pathol Lab Med 2017;141:1544-1557.

14 Zhang Q, Xu M, Verriotto JD, Li Y, Wang H, Gan L, Lam BL, Chen R: Next-generation sequencing-based molecular diagnosis of 35 Hispanic retinitis pigmentosa probands. Sci Rep 2016;6:32792.

15 Valencia CA, Husami A, Holle J, Johnson JA, Qian Y, Mathur A, Wei C, Indugula SR, Zou F, Meng H, Wang L, Li X, Fisher R, Tan T, Hogart Begtrup A, Collins K, Wusik KA, Neilson D, Burrow T, Schorry E, Hopkin R, Keddache M, Harley JB, Kaufman KM, Zhang K: Clinical Impact and Cost-Effectiveness of Whole Exome Sequencing as a Diagnostic Tool: A Pediatric Center's Experience. Front Pediatr 2015;3:67.

-16 Birtel J, Eisenberger T, Gliem M, Muller PL, Herrmann P, Betz C, Zahnleiter D, Neuhaus C, Lenzner S, Holz FG, Mangold E, Bolz HJ, Charbel Issa P: Clinical and genetic characteristics of 251 consecutive patients with macular and cone/cone-rod dystrophy. Sci Rep 2018;8:4824.

17 Lin Y, Gao H, Chen C, Zhu Y, Li T, Liu B, Ma C, Jiang H, Li Y, Huang Y, Wu Q, Li H, Liang X, Jin C, Ye J, Huang $\mathrm{X}$, Lu L: Clinical and next-generation sequencing findings in a Chinese family exhibiting severe familial exudative vitreoretinopathy. Int J Mol Med 2018;41:773-782.

18 Xiao H, Guo Y, Yi J, Xia H, Xu H, Yuan L, Hu P, Yang Z, He Z, Lu H, Deng H: Identification of a Novel Keratin 9 Missense Mutation in a Chinese Family with Epidermolytic Palmoplantar Keratoderma. Cell Physiol Biochem 2018;46:1919-1929.

19 Zhou Q, Cheng J, Yang W, Tania M, Wang H, Khan MA, Duan C, Zhu L, Chen R, Lv H, Fu J: Identification of a novel heterozygous missense mutation in the CACNA1F gene in a chinese family with retinitis pigmentosa by next generation sequencing. Biomed Res Int 2015;2015:907827.

20 Fu J, Li L, Lu G: Relationship between microdeletion on Y chromosome and patients with idiopathic azoospermia and severe oligozoospermia in the Chinese. Chin Med J 2002;115:72-75.

21 Fu S, Cheng J, Wei C, Yang L, Xiao X, Zhang D, Stewart MD, Fu J: Development of diagnostic SCAR markers for genomic DNA amplifications in breast carcinoma by DNA cloning of high-GC RAMP-PCR fragments. Oncotarget 2017;8:43866-43877. 


\section{Cellular Physiology Cell Physiol Biochem 2018;51:2445-2455 and Biochemistry \begin{tabular}{l|l} 
DOI: 10.1159/000495901 & $\begin{array}{l}\text { O } 2018 \text { The Author(s). Published by S. Karger AG, Basel } \\
\text { muwwkarger.com/cpb }\end{array}$
\end{tabular}

Yang et al.: A Novel FZD4 Variant and FEVR

-22 Fu Q, Xu M, Chen X, Sheng X, Yuan Z, Liu Y, Li H, Sun Z, Li H, Yang L, Wang K, Zhang F, Li Y, Zhao C, Sui R, Chen R: CEP78 is mutated in a distinct type of Usher syndrome. J Med Genet 2017;54:190-195.

-23 Li H, Durbin R: Fast and accurate short read alignment with Burrows-Wheeler transform. Bioinformatics 2009;25:1754-1760.

24 Challis D, Yu J, Evani US, Jackson AR, Paithankar S, Coarfa C, Milosavljevic A, Gibbs RA, Yu F: An integrative variant analysis suite for whole exome next-generation sequencing data. BMC bioinformatics 2012;13:8.

25 Psaty BM, O’Donnell CJ, Gudnason V, Lunetta KL, Folsom AR, Rotter JI, Uitterlinden AG, Harris TB, Witteman JC, Boerwinkle E, Consortium C: Cohorts for Heart and Aging Research in Genomic Epidemiology (CHARGE) Consortium: Design of prospective meta-analyses of genome-wide association studies from 5 cohorts. Circ Cardiovasc Genet 2009;2:73-80.

-26 Genomes Project C, Abecasis GR, Altshuler D, Auton A, Brooks LD, Durbin RM, Gibbs RA, Hurles ME, McVean GA: A map of human genome variation from population-scale sequencing. Nature 2010;467:1061-1073.

27 Wang K, Li M, Hakonarson H: ANNOVAR: functional annotation of genetic variants from high-throughput sequencing data. Nucleic Acids Res 2010;38:e164.

28 Tennessen JA, Bigham AW, O’Connor TD, Fu W, Kenny EE, Gravel S, McGee S, Do R, Liu X, Jun G, Kang HM, Jordan D, Leal SM, Gabriel S, Rieder MJ, Abecasis G, Altshuler D, Nickerson DA, Boerwinkle E, Sunyaev S, Bustamante CD, Bamshad MJ, Akey JM, Broad GO, Seattle GO, Project NES: Evolution and functional impact of rare coding variation from deep sequencing of human exomes. Science 2012;337:64-69.

29 Imani S, Cheng J, Mobasher-Jannat A, Wei C, Fu S, Yang L, Jadidi K, Khosravi MH, Mohazzab-Torabi S, Shasaltaneh MD, Li Y, Chen R, Fu J: Identification of a novel RPGRIP1 mutation in an Iranian family with leber congenital amaurosis by exome sequencing. J Cell Mol Med 2018;22:1733-1742.

-30 Fu J, Ma L, Cheng J, Yang L, Wei C, Fu S, Lv H, Chen R, Fu J: A novel, homozygous nonsense variant of the CDHR1 gene in a Chinese family causes autosomal recessive retinal dystrophy by NGS-based genetic diagnosis. J Cell Mol Med $2018 ; 22: 5662-5669$.

-31 Marchler-Bauer A, Bo Y, Han L, He J, Lanczycki CJ, Lu S, Chitsaz F, Derbyshire MK, Geer RC, Gonzales NR, Gwadz M, Hurwitz DI, Lu F, Marchler GH, Song JS, Thanki N, Wang Z, Yamashita RA, Zhang D, Zheng C, Geer LY, Bryant SH: CDD/SPARCLE: functional classification of proteins via subfamily domain architectures. Nucleic Acids Res 2017;45:D200-D203.

-32 Imani S, Ijaz I, Shasaltaneh MD, Fu S, Cheng J, Fu J: Molecular genetics characterization and homology modeling of the CHM gene mutation: A study on its association with choroideremia. Mutat Res 2018;775:39-50.

33 Sizmaz S, Yonekawa Y, M TT: Familial Exudative Vitreoretinopathy. Turk J Ophthalmol 2015;45:164-168.

-34 Takahashi M, Yokoi T, Katagiri S, Yoshida-Uemura T, Nishina S, Azuma N: Surgical treatments for fibrous tissue extending to the posterior retina in eyes with familial exudative vitreoretinopathy. Jpn J Ophthalmol 2018;62:63-67.

35 Fei P, Zhu X, Jiang Z, Ma S, Li J, Zhang Q, Zhou Y, Xu Y, Tai Z, Zhang L, Huang L, Yang Z, Zhao P, Zhu X: Identification and functional analysis of novel FZD4 mutations in Han Chinese with familial exudative vitreoretinopathy. Sci Rep 2015;5:16120.

-36 Kaykas A, Yang-Snyder J, Heroux M, Shah KV, Bouvier M, Moon RT: Mutant Frizzled 4 associated with vitreoretinopathy traps wild-type Frizzled in the endoplasmic reticulum by oligomerization. Nat Cell Biol 2004;6:52-58.

-37 Xu Q, Wang Y, Dabdoub A, Smallwood PM, Williams J, Woods C, Kelley MW, Jiang L, Tasman W, Zhang K, Nathans J: Vascular development in the retina and inner ear: control by Norrin and Frizzled-4, a highaffinity ligand-receptor pair. Cell 2004;116:883-895.

-38 Zhang C, Lai MB, Khandan L, Lee LA, Chen Z, Junge HJ: Norrin-induced Frizzled4 endocytosis and endolysosomal trafficking control retinal angiogenesis and barrier function. Nat Commun 2017;8:16050.

-39 Wei C, Yang L, Cheng J, Imani S, Fu S, Lv H, Li Y, Chen R, Leung EL, Fu J: A novel homozygous variant of GPR98 causes usher syndrome type IIC in a consanguineous Chinese family by next generation sequencing. BMC Med Genet 2018;19:99. 